\title{
Lucro S/A: um CDA para a Administração Financeira
}

\author{
Richard N. Silva1, Thiago G. Mendes', André B. Trombetta', Guilherme T. \\ Schneider', João B. Mossmann'1, Marta R. Bez ${ }^{1}$, Vitor C. S. Valadares ${ }^{1}$ \\ ${ }^{1}$ LOA - Laboratório de Objetos de Aprendizagem - Universidade Feevale \\ ERS 239, 2755 - 93.600-000 - Novo Hamburgo - RS - Brasil \\ richard.n.silva@gmail.com, thiagogmefeevale.br, \{trombetta, \\ gtschneider, mossmann, martabez, vitorvaladaresbr\}@gmail.com,
}

\begin{abstract}
This article has the objective of exposing the development process of the Digital Learning Construct called as Lucro S/A, produced by the Learning Objects Laboratory in association with Applied Social Sciences Institute of Feevale University. Will be introduced auctor who defend the application of the elements used by entertainment games industry in their similar of the education area. Examples of other project using the same development methodology will be given, contextualizing they production process.
\end{abstract}

Resumo. $O$ presente artigo tem o objetivo de expor o processo de desenvolvimento do Construto Digital de Aprendizagem (CDA) Lucro S/A, produzido numa parceria entre o Laboratório de Objetos de Aprendizagem e o Instituto de Ciências Sociais Aplicadas da Universidade Feevale. Serão introduzidos autores que defendem a utilização de elementos da indústria de jogos para entretenimento em seus similares na educação. Serão dados exemplos de outros projetos que utilizaram a mesma metodologia de construção de CDAs aplicada ao construto em questão.

\section{Introdução}

Cada vez mais se verifica o uso de tecnologias digitais, em especial os games, como ferramentas de apoio de aquisição, construção e retenção de conhecimento. A questão está presente em diversas áreas do conhecimento. Das ciências exatas, como a matemática e a física, até aquelas consideradas mais subjetivas, como as artes visuais e a filosofia, se constituindo num campo de pesquisa com ampla atividade.

Com a consolidação da Internet como catalizador do acesso a muitos tipos de informações, a popularização do computador doméstico e, consequentemente, o natural amadurecimento das gerações que nasceram em contato com este tipo de tecnologia, e que recebe rótulos como "Screenagers", "nativos digitais", "Geração M" entre outros (Alves, 2008), a forma como as pessoas buscam, acessam informações e, eventualmente, aprendem novos conhecimentos mudou radicalmente.

Entre todas as tecnologias digitais, os jogos vêm chamando a atenção dos pesquisadores por diversos motivos - pelo seu poder sedutor, por seus aspectos 


\section{CBIE-LACLO 2015}

Anais dos Workshops do IV Congresso Brasileiro de Informática na Educação (CBIE 2015)

simulacionais, pela sua capacidade narrativa não linear, ou mesmo pelo seu altíssimo potencial imersivo (Pinheiro e Branco, 2006). Da mesma forma, o despertar de um particular interesse por parte de pesquisadores de diferentes áreas - da comunicação, da tecnologia da informação, do design e da educação - aponta para uma produção crescente de massa crítica nesta área nas universidades do Brasil e do exterior (Pinheiro e Branco, 2006).

Assim, os aspectos simulacionais presentes nos jogos permitem um processo exploratório baseado numa relação entre estímulo e resposta, a partir de objetos com modelos de comportamento pré-determinados que produzam diferentes significados à medida que o jogador modifica sua ação (Frasca, 2003). Se for realizada uma relação com a estrutura de conhecimentos de Ausubel (2010), é possível supor que o jogador consegue relacionar cada novo resultado da interação nos objetos e seus modelos de comportamento, apresentados por Frasca (2003), a um conhecimento prévio presente em sua estrutura cognitiva, e isso facilita à produção de significado (Ausubel, 2010).

Os jogos digitais são, invariavelmente, simuladores que proporcionam algum tipo de experiência em ambiente controlado (Frasca, 2003). Aqueles que possuem seu foco voltado ao entretenimento exploram camadas narrativas e de jogabilidade visando uma aderência voluntária do jogador (Branco 2011). Essa aderência pode ser utilizada como uma forma persuasiva de manter um jogador/aluno focado na experiência de jogo em produtos orientados a construção de conhecimentos (McGonigal, 2011). No entanto, para que esta experiência seja adequadamente construída, é fundamental que haja uma relação entre a estratégia pedagógica, utilizada na abordagem do conteúdo, e as métricas de game design, que se preocupa, sobretudo, com a forma de entrega de conteúdo e a experiência do usuário, de forma que o resultado produzido seja fruto da sinergia destes dois elementos (Prensky, 2001).

Estabelecer este tipo de sinergia, entre as métricas de game e as estratégias pedagógicas, no entanto, não é facilmente realizável. A maior dificuldade encontra-se no fato de que game designers e educadores detém conjuntos de conhecimentos distintos. Suas abordagens no processo de criação de jogos são diferentes (Prensky 2001).

\section{Jogos para Entretenimento e Jogos Educacionais}

É do senso comum que jogos produzidos com objetivos educacionais e finalidades pedagógicas carecem de qualidade no que se refere a acabamento, divertimento e engajamento. Isso ocorre porque há uma diferença de percepção do foco de produção destes jogos digitais. A percepção parece ser a de que os objetos de aprendizagem, comparados com obras como World of Warcraft, AngryBirds e outros, não tem a mesma qualidade de produção e oferta de divertimento dos últimos (Branco, et al. 2013).

Essa dicotomia ocorre porque há uma preocupação dos desenvolvedores de jogos com os aspectos persuasivos e sedutores dos games e, em contrapartida, uma preocupação dos educadores com a quantidade de conteúdo entregue e com a clareza dessa entrega. Alves reforça esse panorama quando problematiza: 


\title{
CBIE-LACLO 2015
}

Anais dos Workshops do IV Congresso Brasileiro de Informática na Educação (CBIE 2015)

\begin{abstract}
A dificuldade se instaura quando desenvolvedores precisam dialogar com os pedagogos, já que pensam de forma diferenciada. Os primeiros acreditam que o entretenimento pode contribuir para aprendizagem em contraponto, os docentes acreditam que as narrativas devem ter um enfoque em conteúdos escolares. "Jogos para aprender e jogos para divertir", como se não fosse possível articular esses dois prazeres (Alves, 2008, p. 4).
\end{abstract}

Prensky, há 14 anos atrás, defendia que os avanços obtidos pela indústria do entretenimento na produção de jogos de alta qualidade não correspondiam da mesma forma nos produtos voltados ao ensino (2001). Atualmente, esse panorama não sofreu alterações significativas. Apesar da inegável necessidade latente da utilização desse tipo de recurso no processo educativo, bem como da abundante disponibilidade de recursos tecnológicos gratuitos ou de baixo custo financeiro para sua produção, o distanciamento destas duas realidades continua um tema atual.

\section{Aproximação de Horizontes}

Buscando aproximar os dois mundos: jogos para entretenimento e jogos educacionais, foi criado, em 2011 na Universidade Feevale, o Laboratório de Objetos de Aprendizagem (LOA) (Bez et al., 2012). Este é um laboratório que reúne a expertise de profissionais das mais variadas áreas como jogos digitais, design, informática e pedagogia.

A função do LOA é o desenvolvimento de Construtos Digitais de Aprendizagem (CDA) (Branco et al., 2013). Para isso, o grupo atua em parceria com professores de áreas específicas, detentores dos conteúdos que serão incorporados nos CDA's. Estes CDA's são usados como ferramentas de apoio à sala de aula, bem como ao ensino a distância.

Os CDA's são construídos utilizando uma metodologia de desenvolvimento própria, buscando a melhor relação entre conteúdo, acabamento e engajamento, com frequentes medições de resultados.

Foram construídos, desde o surgimento do LOA, cinco construtos digitais: Introdução aos Estudos Virtuais (Valadares et al, 2014), Alien Factory, Beer Game, Desafio Quiz (Fardin et al, 2014) e Zell.

Pelas avaliações recebidas na aplicação dos CDAs em uso na Universidade Feevale, pode-se inferir que é possível unir estes dois mundos: jogos para entretenimento e jogos educacionais. Essa união vem se provando, não só viável, mas eficiente, divertida e envolvente, aumentando o tempo de permanência do aluno no jogo.

A gradual experiência, adquirida pela equipe do LOA ao longo dos projetos anteriores, resultou em um novo CDA, intitulado Lucro S/A. Este CDA é o sexto desenvolvido no LOA e será apresentado a seguir. 
CBIE-LACLO 2015

Anais dos Workshops do IV Congresso Brasileiro de Informática na Educação (CBIE 2015)

\section{O CDA Lucro S/A}

O CDA Lucro S/A, foi desenvolvido no Laboratório de Objetos de Aprendizagem (LOA) para ser utilizado em disciplinas de pós-graduação em Administração Financeira da Universidade Feevale.

A necessidade do jogo veio a partir da percepção de que os alunos que chegam ao fim do curso não colocam em prática todos os conteúdos necessários para a administração de uma empresa. O método, utilizado anteriormente, partia de uma planilha desenvolvida no Microsoft Excel para simular as atividades de administração. Isso resultava em uma dificuldade extra de entendimento dos alunos, uma vez que, além de terem de lidar, com um grande volume de conteúdo, deveriam aprender as particularidades dessa planilha.

O desenvolvimento do CDA foi dividido em três etapas: a primeira constitui-se de uma tradução do atual modelo em Excel, a qual consiste de uma tabela com mais de 50 dados representados, para uma visualização interativa, utilizando a ferramenta de desenvolvimento de jogos digitais Unity; a segunda, atualmente em aplicação, busca remover a dificuldade relatada de lidar com uma quantidade grande de informações, utilizando-se, para isso, dados intermediários e dividindo as etapas de entrega de informações para momento chaves (Figura 1); e uma terceira e última etapa de refinamento e validação do projeto junto ao público-alvo, para aferir se o construto está entregando o conteúdo de maneira esperada pelos conteudistas.

Durante a primeira etapa, julgou-se necessário uma transposição do conteúdo da planilha original para a ferramenta de desenvolvimento de jogos. O objetivo foi verificar se a mecânica fundamental incluída na planilha poderia ser reproduzida sem erros no processo de desenvolvimento e de que haveria tempo hábil para a inclusão de todas as variáveis necessárias para que o CDA continuasse abordando os conteúdos os quais os especialistas em conteúdo consideravam fundamentais.

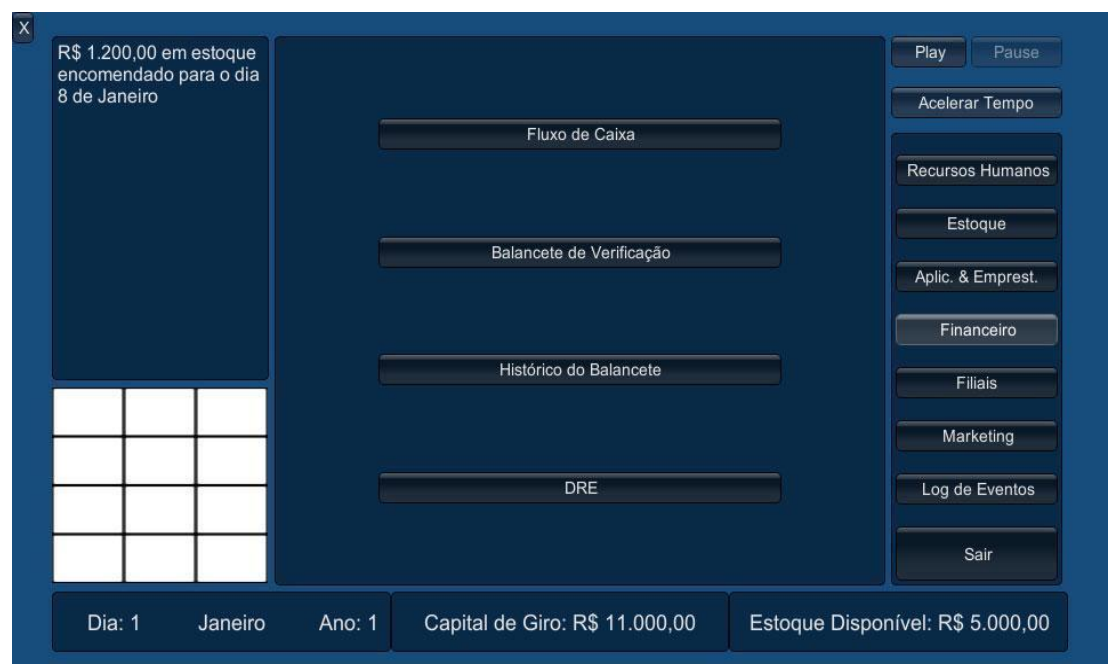

Figura 1. Exemplo de tela após adaptação na Unity (2ª versão).

Após a validação da versão desenvolvida, foi dado início ao processo de transformação do conteúdo em uma experiência jogável, também chamado de 


\section{CBIE-LACLO 2015}

Anais dos Workshops do IV Congresso Brasileiro de Informática na Educação (CBIE 2015)

desenvolvimento de gameplay. Entre os elementos de jogo inseridos no conteúdo, destacam-se as mecânicas relativas ao marketing, a criação de filiais, mecânicas para identificação e ajuste do desempenho dos funcionários e o controle diário da empresa. Este último, no conteúdo original, era feito apenas em período mensal. Além de estabelecer uma experiência envolvente para o jogador-aluno, estes elementos contribuíram para contextualizar suas ações e diversificá-las em diferentes situações.

Ao mesmo tempo, outros itens foram adaptados ou simplificados a fim de diminuir a carga cognitiva do usuário frente à quantidade de informações simultâneas a serem administradas. Alguns exemplos de adaptações incluem a junção de custo de máquinas, móveis e computadores em um único item chamado "Ativo Imobilizado" e a simplificação do cálculo de CMV e Impostos. Foi também durante essa etapa que um ajuste preliminar de impacto referente à tomada de decisões do jogador, conhecida como "balanceamento", foi feito em conjunto com o conteudista, de modo a permitir que o jogo se aproximasse mais da realidade, porém ainda sim mantendo um certo nível de abstração.

Com as mecânicas básicas funcionando teve início a segunda etapa, de adaptação da apresentação dos dados ao usuário. Um estudo prévio de jogos com a temática de administração e gerenciamento de recursos (Figura 2) levou à conclusão de que a interface teria um papel fundamental na compreensão e na usabilidade do jogo, visto que ela auxilia na distribuição e agrupamento de informações, dados e variáveis, contextualizando estes dados.

Optou-se então por uma estética minimalista, auxiliada pelo uso de iconografia marcante e de fácil leitura, onde cada "macro categoria" de dados receberia um ícone próprio. Isso tornou-se necessário para diminuir a carga cognitiva do jogador, visto que ele precisa acompanhar simultaneamente diversos dados e variáveis.

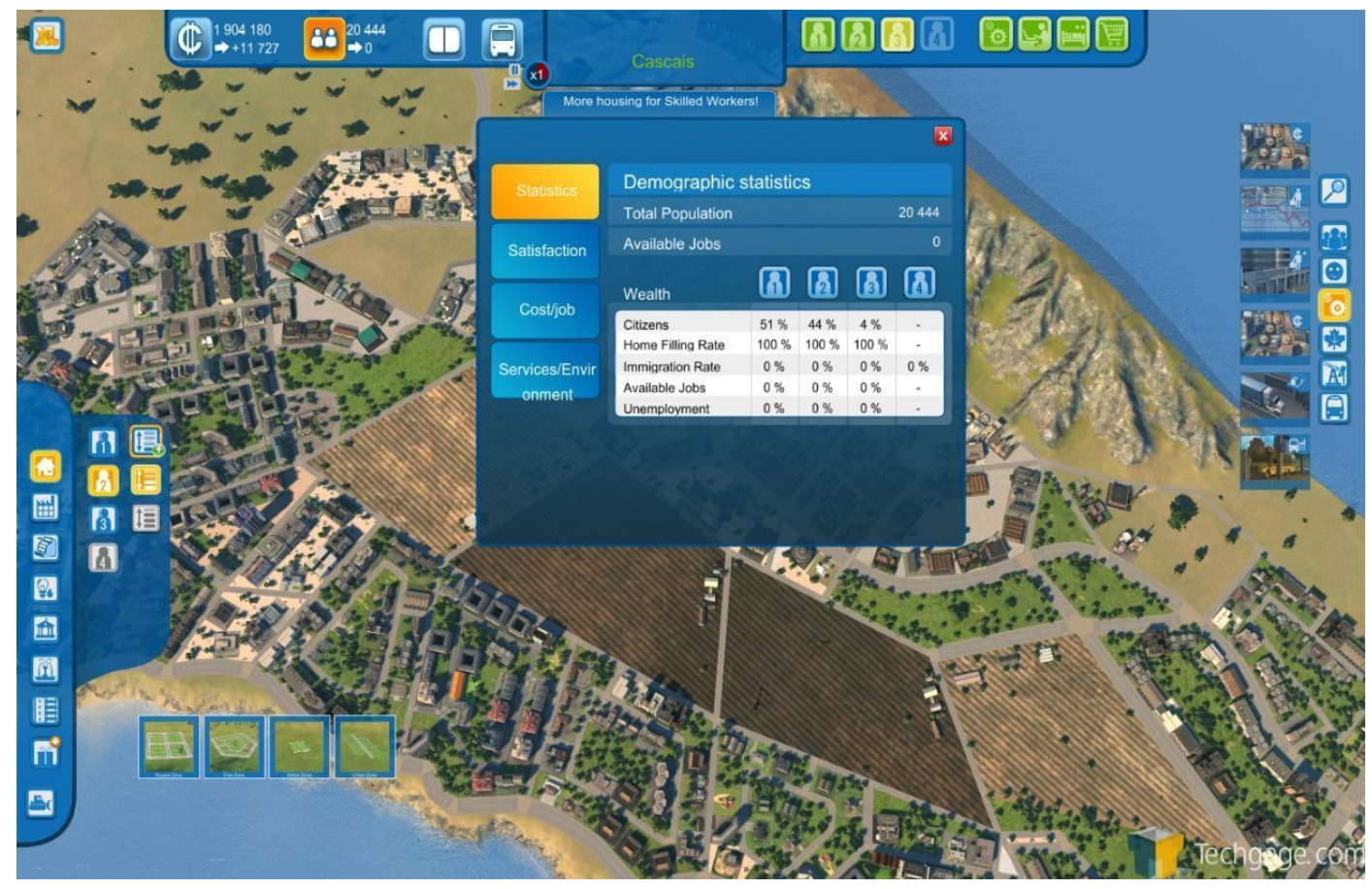

Figura 2. Cities XL: Jogo de administração de cidades 


\section{CBIE-LACLO 2015}

Anais dos Workshops do IV Congresso Brasileiro de Informática na Educação (CBIE 2015)

Além disso, a paleta de cores teve um papel importante na construção da interface. Levou-se em consideração o tema "escritório" e "finanças" durante a escolha das tonalidades. O azul, utilizado como base é utilizada para transmitir equilíbrio e calma (CORTES, 2009). Os tons auxiliares foram escolhidos para facilitar a leitura de dados quantitativos e qualitativos. Vermelho para indicar valores ou condições desfavoráveis, amarelo para representar cuidado ou mudanças de valores/condições e, por fim, a cor verde para indicar valores ou condições favoráveis ao jogador. Estas três cores são utilizadas comumente para representar condições de alerta, atenção e positividade, respectivamente (CORTES, 2009). Além disso, por serem constantemente usadas em planilhas e tabelas financeiras, acabaram sendo incorporadas à paleta de cores.

Tendo em vista o objetivo de diminuição da carga cognitiva foram feitas adaptações da distribuição de conteúdo nas telas já existentes. É possível exemplificar a tela de configuração de jogo. Nela o jogador deve inserir informações e os dados financeiros iniciais de sua empresa e do cenário em que o jogo ocorrerá, tais como o saldo inicial, taxa de juros e crédito disponível. Inicialmente, essas informações tinham de ser alimentadas ao longo de 3 telas diferentes, de modo que cada uma delas possuía um número excessivo de dados e informações, dificultando a compreensão e tomada de decisões.

Outra adição na camada de jogo foi a de gerenciamento e visualização do escritório e do quadro de funcionários. Ao invés do jogador visualizar apenas uma planilha com variáveis mudando constantemente de valor, o CDA foi contextualizado com um cenário que evolui juntamente com o crescimento da empresa. Exemplos disso são os equipamentos adquiridos, funcionários contratados e mudanças de estoque. Todas essas alterações são representadas no ambiente 3D onde ocorre o jogo. Cabe ressaltar que os assets 3D foram desenvolvidos levando em consideração os padrões estéticos e de cores utilizados na interface (Figura 3).

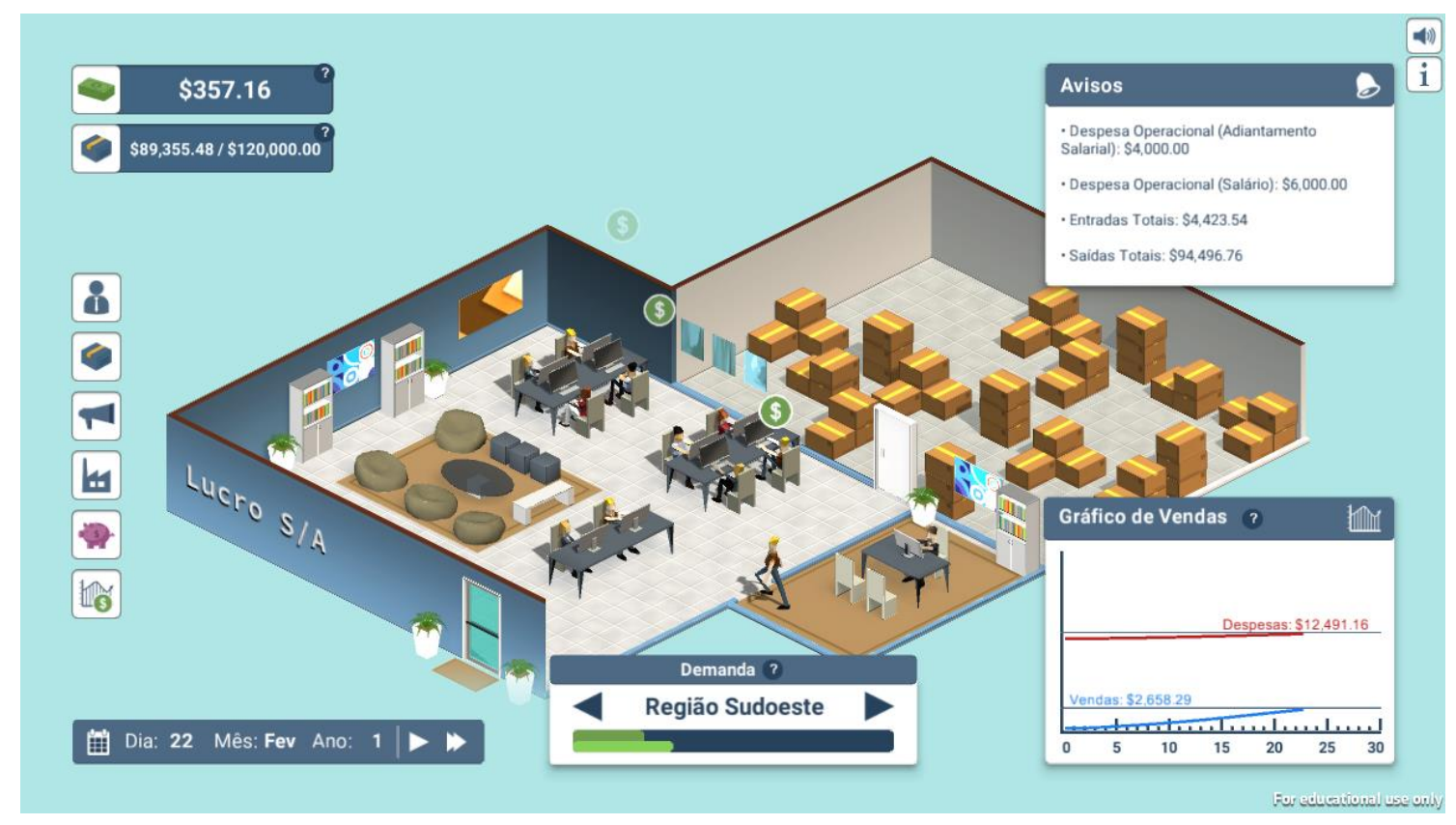

Figura 3. Lucro S/A no seu estágio atual de desenvolvimento 


\section{CBIE-LACLO 2015}

Anais dos Workshops do IV Congresso Brasileiro de Informática na Educação (CBIE 2015)

Após a conclusão dessa etapa do desenvolvimento, teve início a terceira fase, que compreende a validação do CDA junto ao público-alvo. Testes preliminares foram realizados com 33 alunos da disciplina de Administração e Avaliação de Projetos. Essa avaliação teve como objetivo validar o CDA em seu primeiro contato com o público alvo afim de encontrar eventuais necessidades de calibração.

O protocolo de teste consistiu em o professor responsável pela disciplina introduzir o CDA e seus objetivos para assim os alunos então serem submetidos ao teste. Após os alunos testarem o CDA é passado a eles um questionário que tem como objetivo traçar o perfil dos alunos quanto a sua familiaridade com jogos em geral e também sua opinião quanto a eficiência do CDA aplicado em relação aos conteúdos abordados na disciplina, é importante destacar que os alunos testaram o jogo em pequenos grupos, sendo assim o total de respostas obtidas com o questionário é de $13 \mathrm{e}$ não 33, como o número total de alunos. Durante a sessão de teste existia ainda em sala de aula a presença de desenvolvedores do CDA com o objetivo de captar qualquer dificuldade, comentário ou comportamento que não fosse possível obter através da análise dos questionários.

Após uma revisão das repostas obtidas com os questionários foi possível observar que embora mais da metade da turma admita não ter o costume de jogar nenhum tipo de jogo (53\% não jogam qualquer tipo de jogo), todos concordaram que o CDA se apresenta como uma ferramenta motivacional para a aprendizagem. Também $85 \%$ dos alunos concordaram que o CDA permite que o aluno submetido a ele retenha conhecimento adquiro durante a sessão de teste do CDA.

Os relatos coletados durante a sessão de teste em sala de aula trouxeram uma séria de questões relevantes para o refinamento da versão atual do construto. Os comentários recebidos vão desde informações sobre como aplicar e apresentar a atividade para os alunos a melhorias no próprio jogo. As mudanças que influenciaram o desenvolvimento do jogo foram de interface e balanceamento, como criar novas opções de marketing, de nomenclaturas, alguns nomes de alguns atributos utilizados não estavam de acordo e foram corrigidos pelo professor conteudista, e a criação de um tutorial para o construto, com objetivo de tornar o processo de aprendizado do jogo mais simples e diminuir a necessidade de um professor para instruir como utilizar o CDA.

\section{Considerações Finais}

O distanciamento entre a indústria de jogos voltados ao entretenimento e a indústria de jogos educacionais e objetos de aprendizagem ainda é um tema presente e atual. As diferenças nos tipos de produções destas duas categorias ainda são percebidas e, frequentemente alvo de debates. A criação do Laboratório de Objetos de Aprendizagem da Universidade Feevale, foi pautada, sobretudo, pela aproximação destas duas realidades. Com o objetivo de articular, de forma multidisciplinar, as diferentes perspectivas dos profissionais envolvidos e oriundos de diferentes áreas, o resultado tem sido jogos educacionais com alto nível de engajamento e envolvimento. O CDA Lucro $\mathrm{S} / \mathrm{A}$ é mais um exemplo dessa realidade.

Ficou evidente ao longo do desenvolvimento do projeto de que o conteúdo original utilizado em sala de aula possuía uma carga cognitiva elevada e consequentemente uma baixa atratividade. Tendo em vista essa característica, o CDA foi sendo desenvolvido para melhor transmitir as informações ao aluno. Isso foi possível 


\section{CBIE-LACLO 2015}

Anais dos Workshops do IV Congresso Brasileiro de Informática na Educação (CBIE 2015)

principalmente graças à redistribuição de informações e utilização de uma paleta de cores e iconografia adequada.

A maior dificuldade enfrentada no processo de criação foi a etapa de apropriação e assimilação do conteúdo por parte da equipe de desenvolvimento. Só com o conhecimento assimilado é que as devidas adaptações poderiam ser realizadas. O desenvolvimento da experiência de jogo, a partir de uma série de métodos comumente utilizados na indústria dos jogos digitais, proporcionaram um maior nível de envolvimento e tornaram a experiência de aquisição de conteúdo e aprendizagem menos densas e mais divertida.

Por fim, após todas as modificações realizadas no jogo após a coleta de informações no teste preliminar este construto deve se tornar parte integrante das disciplinas da Universidade no início do segundo semestre de 2015.

\section{Bibliografia}

Alves, Lynn Rosalina. 2008. "Relações entre os jogos digitais e aprendizagem: delineando percurso.” Educação, Formação \& Tecnologias 1 (2): 3-10.

Ausubel, David P. 2010. The Acquisition and Retention of Knowledge.Boston: Kluwer Academic Publishers.

Branco, Marsal A. A., Marta R. Bez, João B. Mossmann, e Thiago G. Mendes. 2013. "Dimensões dos Jogos de Ensino." INTERNATIONAL CONFERENCE ON ENGINEERING AND COMPUTER EDUCATION 1: 41-51.

Branco, Marsal A.A. 2011. "Jogos Digitais: Metodologia e Conceitos para uma mídia indisciplinada.” Unisinos, São Leopoldo.

Cortes, Maria Claudia. Color in Motion. Rochester: 2003. Disponível em: $<$ http://www.mariaclaudiacortes.com> Acesso em: 27 ago. 2015.

Frasca, Gonzalo. 2003. "Simulation versus Narrative: Introduction to Ludology.” Em The Videogame Theory reader, por Mark J. P. Wolf e Bernard Perron. New York: Routledge.

McGonigal, Jane. 2011. Reality is Broken. New York: Penguin Press.

Pinheiro, Cristiano M.P., e Marsal A.A. Branco. 2006. "Uma Tipologia dos Games." Sessões do Imaginário 11 (15): 33-39.

Prensky, Marc. 2001. Digital Game-Based Learning. St. Paul: Paragon House. 